\title{
Die Stratigraphie der weichselzeitlichen Ablagerungen der Niederlande und Belgiens
}

\author{
Von Waldo Zagwijn, Haarlem und Roland Paepe, Brüssel
}

Mit 6 Abbildungen

$\mathrm{Z} u \mathrm{~s}$ a $\mathrm{m}$ men $\mathrm{f}$ a s sung. Es wird der lithostratigraphische Aufbau der niederländischen und belgischen weichselzeitlichen Schichten behandelt und korreliert. Die in den Niederlanden im Flugdecksand gewonnene Klimastratigraphie auf biostratigraphischer Grundlage konnte dadurch auf das Übergangs- und Lößgebiet Belgiens übertragen werden. Es ergeben sich dadurch Verknüpfungen mit dem mitteleuropäischen Lößgebiet.

$\mathrm{Su} \mathrm{m} \mathrm{m}$ a r y. The lithostratigraphy of the Weichselian (Last Glacial) beds of the Netherlands and Belgium is discussed and a correlation has been established. The climatic stratigraphy obtained in the coversand area of the Netherlands and based mainly on biostratigraphic data, could now be applied to the transitional and loessarea of Belgium. This opens a possibility for connecting the loess stratigraphy of Central Europe and the Alpine region with that of the north-westeuropean area.

\section{Einführung}

Der Gliederung der Ablagerungen der letzten Eiszeit ist eine umfangreiche Literatur gewidmet und bekanntlich stehen sich die Meinungen der verschiedenen Autoren schroff gegenüber. Es kann nicht unsere Absicht sein, hier eine Übersicht dieser Auffassungen zu geben, die zwischen der Annahme einer ungegliederten Kaltzeit (z. B. BüDEL 1950) und einem recht komplizierten Ablauf mit mehreren Interstadialen (z. B. Gross 1956, 1958, 1964) schwankt.

Es ist unsere Ansicht, daß eine Klima-Gliederung der letzten Eiszeit auf Daten aus jeweils relativ engbegrenzten Gebieten fußen sollte, die eine möglichst vollständige Schichtenfolge aufweisen. Die Konstruktion von Klimakurven durch Vermischung von Daten aus weit auseinanderliegenden Gebieten mit unvollständiger Stratigraphie kann nur zu Fehlschlüssen führen.

Ein nächster Schritt ist die Verknüpfung solcher Gebiete, nicht nur auf Grund der erhaltenen Klimakurven, sondern wenn irgend möglich auch basierend auf lithostratigraphischen, biostratigraphischen und chronologischen Vergleichen. In diesem Aufsatz wird dieser Gedanke weiter ausgeführt auf Grund des Vergleichs der Ergebnisse in den Niederlanden, in geme:nschaftlicher Arbeit veröffentlicht von van DER HAMmen, MaArleveld, Vogel und Zagwijn (1967) und jener in Belgien (Paepe und Vanhoorne 1967).

Vor allem in den Niederlanden sind viele Becken erhalten, die organogene Schichten des letzten Glazials enthalten. So standen viele Pollendiagramme aus fast allen Phasen der Eiszeit, mehr als $30{ }^{14} \mathrm{C}$-Daten für das Pleniglazial und außerdem noch eine gleiche Zahl von ${ }^{14} \mathrm{C}$-Daten für das Spätglazial zur Verfügung.

Andererseits sind die belgischen Studien wichtig für die Verknüpfung der niederländischen, im wesentlichen im Flugdecksandgebiet gewonnenen Stratigraphie mit jener des Lößbereichs (Abb. 1).

In Belgien sind die spätpleistozänen Schichten westlich der Sambre-Maas-Rinne fast zusammenhängend verbreitet. Die Mächtigkeit und Korngröße der Schichten kann von Gebiet zu Gebiet stark schwanken. Dies hat schon seit langer Zeit dazu geführt, in Belgien drei Landschaften zu unterscheiden: die „Lehmlandschaft" im Süden, die „Sandlandschaft" im Norden und dazwischen die nach Osten auskeilende "Sandlehmlandschaft". In mehr genetischer Sprache: das Lößgebiet, das Decksandgebiet und das Übergangsgebiet. Die 

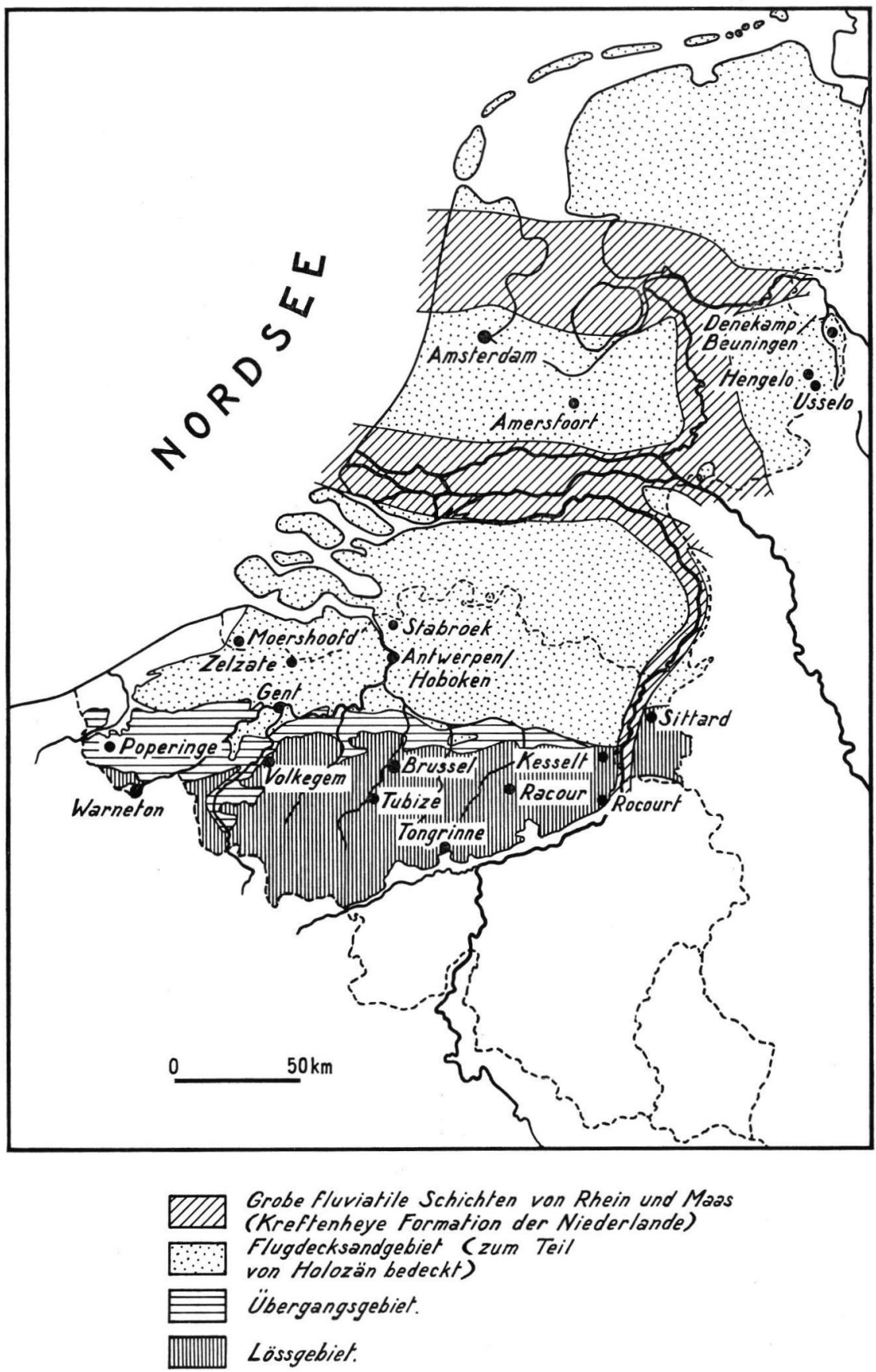

Abb. 1. Verbreitung der spätpleistozänen Ablagerungen in den Niederlanden und Belgien. Wichtige, im Text aufgeführte Aufschlüsse. 
Wichtigkeit des Vorkommens in so begrenztem Raum genetisch ganz verschiedener spätpleistozäner Sedimentationsgebiete, die sich einerseits an die Lößgebiete Mittel- und SüdEuropas anschließen, andererseits an die nordwesteuropäischen Decksandgebiete, dürfte ins Auge springen. Da die beiden Bereiche sich zudem über das Übergangsgebiet hinweg verknüpfen lassen, ist hiermit die Möglichkeit gegeben, die in den Niederlanden gewonnene Klimastratigraphie mit jener der Lößbereiche zu verknüpfen und letzten Endes Vergleiche mit der im alpinen Bereich gewonnenen Erkenntnis anzustellen. Daß die Zahl der Pollendiagramme und ${ }^{14} \mathrm{C}$-Daten im südlicheren Gebiet nur noch gering ist im Verhältnis zu den Niederlanden, liegt in der Natur der Dinge. Eben jene Becken mit zahlreichen organogenen Schichten und wechselvoller Stratigraphie, die dort biostratigraphische und chronologische Studien ermöglichten, werden zum Lößbereich hin spärlicher, um schließlich im eigentlichen Lößgebiet zu verschwinden. Hier bleiben zur Datierung neben der Morphostratigraphie im wesentlichen nur ${ }^{14} \mathrm{C}$-Datierungen an Humusböden übrig, die bekanntlich kaum mehr als Anhaltspunkte für ein minimales Alter der Schichten geben können.

Es lassen sich aber, wie im folgenden ausgeführt werden soll, Verknüpfungen nach lithostratigraphischen Gesichtspunkten durchführen und soweit wie möglich mit den anderen erwähnten Methoden bestätigen. Da aber die lithostratigraphische Gliederung im Grunde, wie sich in den Niederlanden nachweisen ließ, ein Abbild der Klimaentwicklung ist, ist die Möglichkeit der Korrelation über das niederländische Gebiet hinaus ein Argument dafür, daß der dort gewonnenen Klimagliederung eine mehr als örtliche Bedeutung zukommt.

\section{Die Niederlande}

a. Stratigraphie des Flugdecksandgebietes

Die Ablagerungen der letzten Eiszeit, des Weichselians, der Niederlande können in zwei größeren lithostratigraphischen Einheiten gegliedert werden, die Kreftenheye-Formation und die Twenthe-Formation. Zur Kreftenheye-Formation gehören vor allem grobkörnige Sande und Flußschotter des Rheins und der Maas, weiterhin auch gewisse Auelehme und Sande der Flußdünen, die genetisch mit diesen Flußablagerungen verknüpft sind. Andererseits gehören zur Twenthe-Formation alle jene Ablagerungen des Periglazialbereichs, die lokaler Herkunft sind, wie Flugdecksande, Lösse, Beckenlehme, Schmelzwasserablagerungen, Solifluktionsschichten und organogene Schichten. Als fluvioperiglazial werden neuerdings alle jene Periglazialablagerungen lokaler Herkunft bezeichnet, deren Entstehung mit fließendem oder stehendem Wasser in Verbindung gebracht wird, also eben jene Schmelzwasserablagerungen (niveofluviatil), Beckenlehme usw.

Es können zwei Bereiche der Twenthe-Formation unterschieden werden: der Lößbereich im äußersten Süden der Provinz Limburg und das Flugdecksandgebiet der übrigen Niederlande (Abb. 1). Vor allem in (Saale-)glazialen Becken und in Tälern des letzteren Bereichs fanden sich die vollständigsten Profile, die auch organische Schichten enthielten und somit der Pollenanalyse und Radiokarbonmethode zugänglich gemacht werden konnten. Es konnte daher aus einem relativ kleinen Gebiet eine Klimakurve auf biostratigraphischer und chronologischer Grundlage konstruiert werden, wobei eine Vermischung mit Daten anderer Gebiete vermieden wurde.

Obwohl sich sehr klar gezeigt hat, daß die lokale Ausbildung der Schichten stark wechseln kann, außerdem durch Fortfall gewisser Einheiten kleinere oder größere Schichtlücken vorhanden sein können, hat es sich doch auf Grund dieser Profile als möglich erwiesen, eine gewisse Standardlithostratigraphie des Flugdecksandbereichs zu erkennen, eine Folge gewisser charakteristischer Schichtausbildungen, die sich immer wieder erfassen läßt, trotz lokalem Wechsel der einzelnen Lithologien. Für Einzelheiten wird auf die Ar- 
beit von van DER HAMmen et al. (1967) hingewiesen; hier seien nur einige Hauptzüge hervorgehoben.

Der bis $3 \mathrm{~m}$ mächtige Jüngere Flugdecksand des Spätglazials kann wie schon oft be-schrieben zweigeteilt werden. Zwischen den beiden Einheiten findet sich öfters eine charakteristische Bodenbildung, die „Usselo-Schicht“. Es kann aber auch der Altere Flugdecksand, der schon zum Pleniglazial gehört, wie sich jetzt ergeben hat, zweigeteilt werden. Die obere der beiden Schichten, der Altere Flugdecksand II, meistens weniger als $1 \mathrm{~m}$ mächtig, ist sehr charakteristisch. Es handelt sich um einen etwas lehmigen, zur Unterkante hin teilweise auch gröberen Flugsand mit sehr auffallender Horizontalschichtung, die nicht von Kryoturbationen gestört worden ist. Dann folgt eine Steinsohle mit Wüstenlack, die von großen Frostkeilen begleitet wird. Es ist dies die Beuningen-Schicht, die sich über sehr große Flächen verfolgen läßt. An gewissen Orten ist an Sielle der Steinsohle eine etwas mächtigere Kiesschicht fluvioperiglazialer Entstehung vorhanden.

Die Schichten unterhalb der Beuningen-Steinsohle sind meistens stark kryoturbat und können sehr verschiedenen Alters sein. An einigen Stellen aber scheint die Schichtlücke nicht sehr groß zu sein und man findet einen Flugdecksand, der dem Alteren Flugdecksand II sehr ähnlich ist. Es ist dies der Altere Flugdecksand I. Ofters ist diese Schicht durch eine Steinsohle von den unterlagernden fluvioperiglazialen Sanden getrennt. Diese aber ist nicht mit Frostkeilen verknüpft.

Bisher wurde der Altere Flugdecksand (II) als Pleniglazial B bezeichnet. Es scheint daher angebracht, die beschriebene vollständigere Folge unter Einbeziehung der Beuningen-Steinsohle als Obere Pleniglaziale Schichtenreihe zusammenzufassen.

Im Gegensatz dazu findet man in den M it t le ren Pleniglazialen S chicht e n hauptsächlich Schichten fluvioperiglazialer und organogener Entstehung, während Flugdecksande zurücktreten. Die Frequenz organischer Substanzen ist sehr auffallend und bildet einen schroffen Gegensatz zu den Schichten des Oberen Pleniglazials, aus denen bisher trotz bester Aufschlußverhältnisse noch nie organogene Reste bekannt geworden sind, also auch jegliche Möglichkeit zur Radiokarbondatierung fehlt. Die Mächtigkeit schwankt, kann aber viele Meter sein.

Ofters lassen sich im Mittleren Pleniglazial drei lehmige torfhaltige Horizonte, getrennt von niveo-fluviatilen Sanden, die Dryasfloren enthalten können, erkennen. Öfters sind aber auch nur zwei Lehmschichten vorhanden, indem die beiden oberen sich zu einer Lehmschicht vereinigen. Würgeböden sind weit verbreitet und meistens mit den Lehmhorizonten verbunden. Frostkeilhorizonte sind seltener. Ihre Enstehung hängt mit den Sanden zwischen den Lehmen bzw. mit Diskordanzen zwischen Sanden und Lehmen zusammen.

In Flußtälern findet sich öfters nur eine jüngste Lehm- oder Torfschicht und darunter fluviatile Schichten in ziemlicher Mächtigkeit (bis $15 \mathrm{~m}$ ), die sich nur schwer gliedern lassen.

Die Schichten des Unteren Pleniglazials konnten bisher mangels geeigneter Aufschlüsse nur an wenigen Stellen studiert werden. Es handelt sich vor allem um lehmige horizontalgeschichtete Flugdecksande vom Typus des Alteren Flugdecksandes, die völlig frei von organischen Substanzen sind. Sie sind verknüpt mit Frostkeilen, die sehr große Ausmaße erreichen können, z. B. in der Gegend von Amersfoort (Abb. 2).

In vielen Fällen lagern die pleniglazialen Schichten unmittelbar auf Torfen und Tonen des Eem-Interglazials. In gewissen Becken aber, vor allem wieder bei Amersfoort, ist noch eine Reihe frühglazialer Schichten dazwischen geschaltet. Es handelt sich in der Hauptsache um grobe Flugdecksande, die in ihrer Korngröße und Schichtung völlig dem spätglazialen Jüngeren Flugdecksand entsprechen. Zwischengeschaltet sind zwei Torf- und Gyttja- 


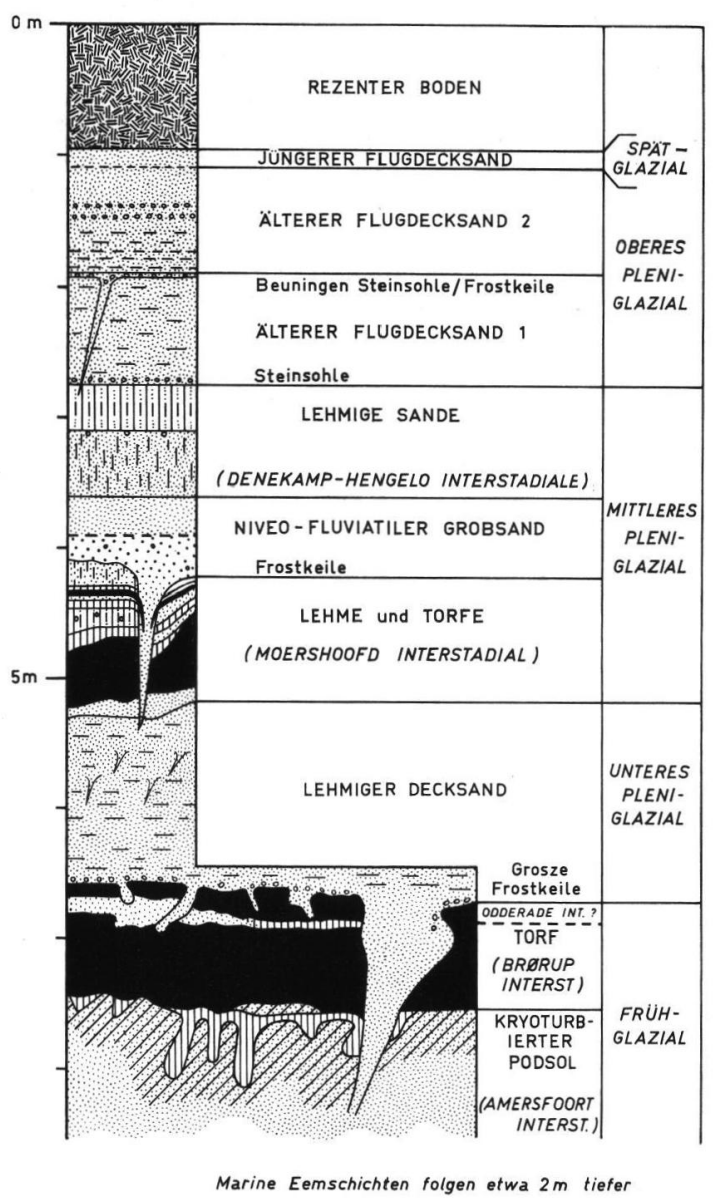

Abb. 2. Profil des Baugruben-Aufschlusses De Liendert bei Amersfoort, Niederlande, (September 1967).

Horizonte, die seitlich in Podsole übergehen. Neuerdings sind auch kryoturbate Störungen innerhalb dieser Schichten beobachtet worden (Abb. 2).

Die Abb. 2 gibt eine Ubersicht der Profilwand einer Baugrube bei Amersfoort, die im Herbst 1967 studiert werden konnte, und mit Ausnahme des Spätglazials alle wesentlichen Schichten des Früh- und Pleniglazials enthält $\left.{ }^{1}\right)$. Abb. 4 faßt die Lithostratigraphie der letzteiszeitlichen Schichten der Twenthe Formation tabellarisch zusammen.

b. Klimageschichte und Chronostratigraphie

Aus dieser Schichtenfolge konnte vor allem mit Hilfe der Pollenanalyse und ${ }^{14} \mathrm{C}-\mathrm{Da}$ tierungen ein ziemlich vollständiges Bild der Klimaentwicklung der letzten Eiszeit in den Niederlanden gewonnen werden. Andererseits war es mit diesen Methoden möglich, unvollständige Profile, die naturgemäß in der Mehrzahl sind, zu deuten und einzustufen (ANDERsen et al. 1960, ZAGWijn 1961, van der Hammen et al. 1967, Vogel und Zagwijn 1967). Für Einzelheiten sei auf die genannten Arbeiten hingewiesen.

1) Ein vorläufiges ${ }^{14} \mathrm{C}$-Datum der Oberkante der als „Moershoofd-Interstadial“ gedeuteten Torfschicht in 4,30 m Teufe ergab rund 45000 Jahre, eine andere von der Unterkante dieser Schicht. bei $5,10 \mathrm{~m}$ ergab mehr als 50000 Jahre. 
Im Frühglazial konnten in den beiden erwähnten Torfhorizonten zwei klimatisch günstigere Phasen nachgewiesen werden, die als Amersfoort und Brørup-Interstadiale bezeichnet wurden. Sie sind beide, wie die pollenanalytischen Untersuchungen ergaben, gekennzeichnet durch völlige Wiederbewaldung der in den kälteren frühglazialen Stadialen I und II entwaldeten Landschaft, in der es zur Bildung von Flugdecksanden kam. Während dieser frühen Interstadiale erreichte die Juli-Mitteltemperatur Werte von $15^{\circ}$ bis $17^{\circ} \mathrm{C}$., während sie in den Kaltphasen unter $10^{\circ} \mathrm{C}$. absank, ohne aber extrem niedrige Werte zu erreichen.

Der in Abb. 2 dargestellte Aufschluß von Liendert-Amersfoort hat außerdem noch Hinweise auf die Existenz eines dritten Interstadials des Früh-Weichselians, nach dem Brørup Interstadial, gegeben, daß mit dem von Averdieck (i. Dr.) für Nordwest-Deutschland nachgewiesenen Interstadial von Odderade übereinstimmen dürfte.

Auch im mittleren Weichselian, im Pleniglazial, konnten Klimaschwankungen pollenanalytisch belegt werden, und zwar handelt es sich dabei um Klimaverbesserungen, während denen es nicht zur Wiederbewaldung kam. Es kommt in den Pollendiagrammen vorübergehend zu einer Ausbreitung der Birke (Strauchbirken), während auch der Gehalt gewisser Wasserpflanzen (z. B. Batrachium) in gewissen Profilen stark ansteigt.

Übrigens bleibt der Nichtbaumpollengehalt hoch, und wir müssen uns die Landschaft als Tundra vorstellen, die sich vorübergehend in eine Strauchtundra verwandelte. Mit Sicherheit konnten zwei solcher Klimaverbesserungen, die auch als Interstadiale bezeichnet werden können, nachgewiesen werden. Eine um 37000-39000 J. v. h., das He n gelo-Interstadial, die andere um 29000-32000 v. h., das DenekampInterstadia 1 . Eine weitere Klimaverbesserung, die sich zwar pollenanalytisch noch nicht eindeutig hat nachweisen lassen, dürfte den Zeitraum rund $43000-50000 \mathrm{~J}$. v. h. umfassen.

Es ist sehr auffallend festzustellen, daß diese Interstadiale den obenerwähnten torfigen Lehmhorizonten des Mittleren Pleniglazials entstammen. Offenbar war das Klima letzten Endes verantwortlich für den Wechsel der Lithologie. In den sandigen Zwischenmitteln findet man denn auch die Beweise für extrem kaltes Klima, vor allem Frostkeile. Es wird die Vermutung geäußert, daß sich in solchen Phasen wenigstens vorübergehend die Tundralandschaft in eine Polarwüste verwandelt hat ${ }^{2}$ ). Die Phase 43000-50000, die nach der Pollenanalyse eine Tundraphase ist, wäre dann zwischen zwei Phasen mit Polarwüste eingeschaltet und als „Interstadial“ zu bezeichnen. Sie wird hier weiterhin als Moershoof d-Interstadial bezeichnet.

$\mathrm{Daß}$ es in unserer Gegend während des Pleniglazials in der Tat zur Bildung einer Polarwüste gekommen sein muß, zeigt sich aber am deutlichsten in der weitverbreiteten Schicht der Beuningen-Steinsohle des Oberen Pleniglazials, die den größten Teil des Zeitraums von 14000-26000 Jahre vor heute umfassen dürfte. Die Anwesenheit einer Polarwüste zur Zeit der stärksten Klimadepression ist verantwortlich für das völlige Fehlen jeglicher Überlieferung organischer Substanz aus diesem Zeitraum.

Es liegt weiterhin die Vermutung nahe, daß sich auch an der Basis des Pleniglazials, im Unteren Pleniglazial, die Zeugen einer analogen Polarwüstenzeit in der Form von Riesenfrostkeilen und einer Steinsohle finden, also älter als 50000 Jahren vor heute. Im Mittleren Pleniglazial hat es solche Phasen dagegen höchstens nur vorübergehend gegeben, vielleicht zwischen 43000 und $39000 \mathrm{~J}$. v. h.

Diese Zeit war vorwiegend durch eine Tundravegetation, die sich vorübergehend in eine Strauchtundra verwandeln konnte, gekennzeichnet. Es ist angenommen worden, daß

2) Unter Polarwüste wird hier mit Aleksandrova (1960) eine fast völlig vegetationsfreie Landschaft des Periglazialbereichs verstanden, wie sie sich heute im nördlichen Teil der Insel NovaZembla findet. Die Juli-Mitteltemperaturen bleiben dort unter $5^{\circ} \mathrm{C}$. Torfbildungen fehlen dieser Landschaft vollständig. 


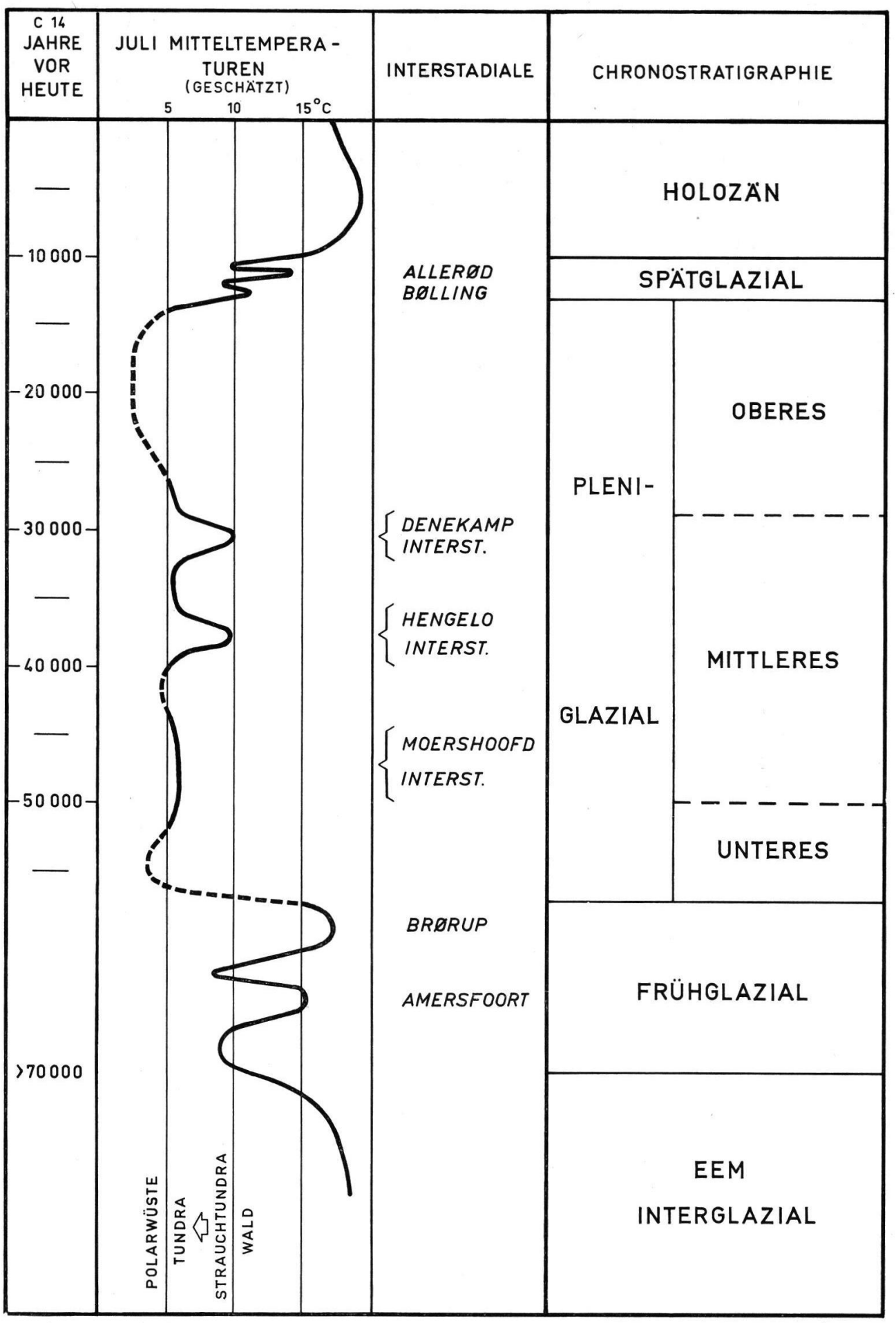

Abb. 3. Klimakurve und Chronostratigraphie der Letzten Eiszeit in den Niederlanden. 
während der Strauchtundrenphasen eine Juli-Mitteltemperatur von $8^{\circ}$ bis $10^{\circ} \mathrm{C}$ und während der Tundrenphasen eine solche von $5^{\circ}$ bis $8^{\circ} \mathrm{C}$ herrschte, dagegen in Zeiten der Polarwüste die Juli-Mittelwerte unter $5^{\circ} \mathrm{C}$ absanken.

So wurde die Klimakurve Abb. 3 konstruiert. Deutlich zeigt sich, daß die Kaltphasen des Früh- und Spätglazials ungefähr die gleichen Temperaturverhältnisse gehabt haben wie die wärmsten Phasen (Hengelo-Denekamp) des Pleniglazials.

Es wird an dieser Stelle weiter darauf verzichtet, auf Einzelheiten der schon längst bekannten Gliederung und Klimaentwicklung des Spätglazials einzugehen (VAN DER Hammen 1951, 1952; de Vries, Barendsen, Waterbolk 1958). Allerdings sei darauf hingewiesen, daß sich die Chronologie nach den heute zur Verfügung stehenden ${ }^{14} \mathrm{C}$ Daten und in guter Übereinstimmung mit anderen Gebieten Europas folgendermaßen gestaltet:

\author{
Ende des späten Dryas-Stadials \\ Allerød-Interstadial \\ Bølling-Insterstadial
}
um $10000 \mathrm{~J} . \mathrm{v} . \mathrm{h}$.
$11000-11800 \mathrm{~J} . \mathrm{v} . \mathrm{h}$.
$12000-12400$ J.v. h.

\title{
III. Belgien
}

Im folgenden werden die in der Einführung erwähnten drei Gebiete behandelt (Abb. 1 und Abb. 4).

a. Das Decksandgebiet

Das Decksandgebiet liegt in der nördlichen Tiefebene Belgiens, die sich durch eine außerordentlich flache Form der Landschaft auszeichnet. Die Gleichmäßigkeit des Reliefs ist sicherlich eine der Ursachen dafür, daß die Stratigraphie der Decksandformation lange unbekannt blieb.

Die Aufschlüsse des Umleitungskanals um Gent und die dortigen Häfen sowie die Tunnelarbeiten in Zelzate (Abb. 5) und Antwerpen haben in hohem Maße dazu beigetragen, diesen Mangel zu beheben. Jene wichtigen Aufschlüsse fanden sich im sogenannten „Flämischen Tal“ (Tavernier 1946), das seine vollständigste Ausbildung in der Gegend nördlich von Gent hat. Meistens lagern dort dem Tertiär „Torf und Kiesschichten “ 3 ) auf. Diese Schichten gehören zum Eem-Interglazial, sowohl auf Grund der paläobotanischen Befunde (PAepe und Vanhoorne 1967) wie an der auf Grund der Anwesenheit von Tapes senescens var. eemiensis (PAEPE 1965) und Corbicula fluminalis.

Diese Formation, in Belgien bekannt unter der Bezeichnung „sables marins à Corbicula fluminalis de l'assise d'Ostende“, wurde von ihrem Entdecker G. Dollfus, 1884, als Alt-Pleistozän bezeichnet, von J. LoRIÉ, 1903, bereits ins Eem gestellt. Dessen ungeachtet ist das Alter lange umstritten gewesen. V. Nordmann (1908) und nach ihm G. Dubois, A. Hacquaert und F. Halet (1931) sprachen sogar von einem postglazialen Alter. Erst auf Anregung R. TAverniers, der anfänglich (1946) ein würminterstadiales Alter verteidigte, wird diese Formation seit 1954 endgültig dem Eem-Interglazial zugewiesen. Ein wichtiges Argument war hierbei, daß in den Tälern der tieferen Landschaften Belgiens die Kiesablagerungen mit Corbicula fluminalis von drei Lößschichten überlagert werden.

In den Aufschlüssen von Gent und in zahlreichen Bohrungen des „Flämischen Tals“ und der Küstenebene sind die Kiesablagerungen meistens ziemlich mächtig und liegen zwischen 5 und 11 Metern unter Ostender Pegel. In Zelzate (Abb. 5) ist die Ablagerung nur geringmächtig, scheint aber in einem unmittelbar anschließenden Aufschluß mit einer teilweise erhaltenen Bodenbildung verknüpt zu sein, die eine Intensität der Verwitterung

3) Die hier und im folgenden jeweils zwischen Anführungszeichen zitierten litho-stratigraphischen Bezeichnungen sind Übersetzungen der von PaEPE (in PAEPE und Vanhoorne 1967) für die Gegend eingeführten Begriffe. (Tabelle Abb. 4.) 


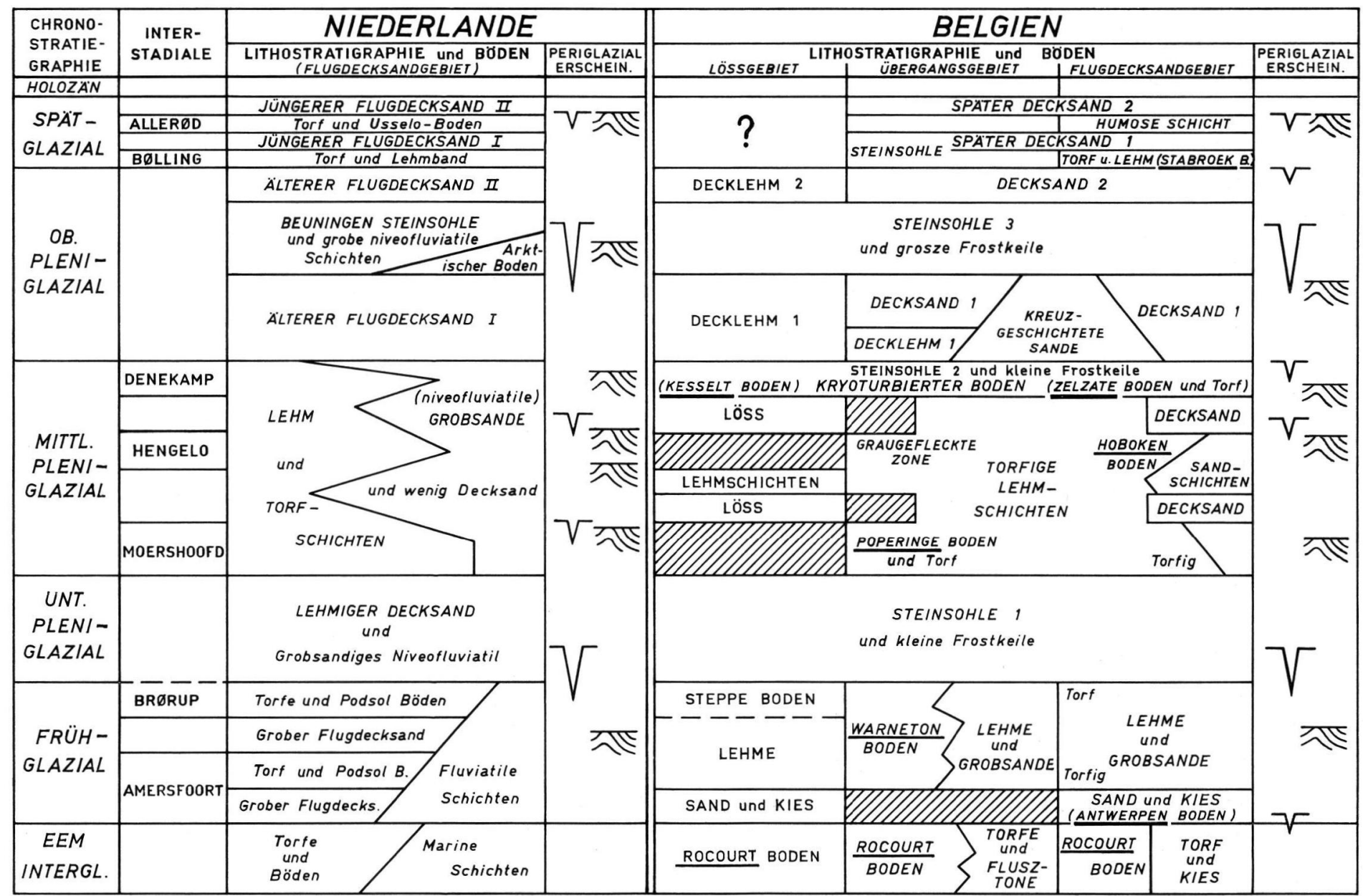

Abb. 4. Vergleich der Lithostratigraphie des Spätpleistozäns der Niederlande und Belgiens. 


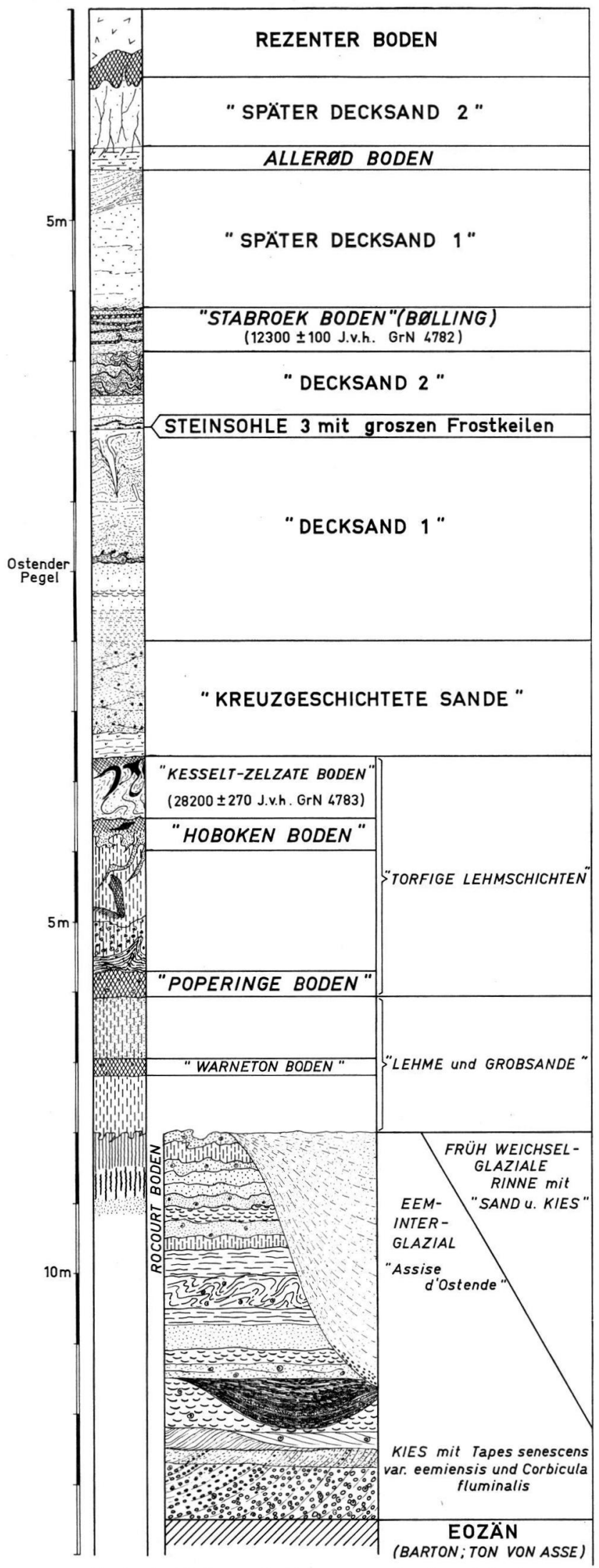

Abb. 5. Profil des Aufschlusses in Zelzate (Belgien). 
zeigt, die sich schwerlich anders als auf warm-feuchte, interglaziale Klimabedingungen zurückführen läßt.

Sowohl diese Bodenbildung als auch die marinen Kiese sind in Zelzate (Abb. 5) durch tiefe Einschnitte, die mit "Sanden und Kiesen“4) gefüllt sind, erodiert. Es folgen lehmige Schichten, die eine unregelmäßige gezackte Abgrenzung an der Unterkante haben. Im unteren Teil fanden sich zwei graue Lehmschichten, die jeweils an der Oberkante einen humosen oder torfigen Horizont mit vielen Landmollusken zeigten. Stellenweise finden sich Sandlinsen und sogar Feuersteinblöcke. Dieser ganze Komplex wurde als „Lehme und Grobsande" 5 ) bezeichnet. Es folgt noch oben eine Reihe von sehr unregelmäßig verlaufenden, stark abwechselnden bleichsandigen und dunklen, lehmigen Bändern mit vereinzelten kleinen Frostkeilen an der Oberkante der Schichtfolge. Dann wird die Fazies wieder lehmiger und kompakter und die Schichten werden wiederum durch einen humosen, diesmal stark kryoturbierten Horizont abgeschlossen. Dies sind die „torfigen Lehmschichten". Es folgt dann ein weiterer, sehr gut erkennbarer und daher sehr wichtiger Horizont, nämlich ein stark kryoturbierter Torf, der sich lückenlos den ganzen Aufschluß hindurch verfolgen ließ. Dieser Horizont wurde auf $28200 \pm 270$ Jahre vor heute datiert (GrN-4783). Um vorgreifen auf spätere Korrelationen zu vermeiden, wurde dieser Horizont als "Z e l z a t e - B o d e n" bezeichnet.

Die "torfigen Lehmschichten" lassen sich in anderen Aufschlüssen gut verfolgen. So z. B. bei Gent (Sifferdok), wo diese Schichten direkt dem Eem auflagern. Sie sind durch eine 4 bis $5 \mathrm{~m}$ mächtige Folge von stark torfigen, wenige Millimeter dicken Lehmbändchen gekennzeinet, die mit dünnen Feinsandlagen abwechseln. Stellenweise sind die letzteren etwas mächtiger, zeigen aber äolischen Charakter und kleine Frostkeile an der Basis.

In Zelzate (Abb. 5) wie in Gent sind die „torfigen Lehmschichten“ bzw. der „ZelzateBoden" erodiert; an ihrer Stelle finden sich breite, seichte Rinnen, die mit fluviatilem Grobsand und Lehmlinsen gefüllt sind und die als „kreuzgeschichtete Sande“ bezeichnet werden. Diese Schichten sind als Schmelzwasserablagerungen anzusprechen, analog den niveofluviatilen Schichten der Niederlande (v. D. HAMmEN 1952). Sie sind von großen Frostkeilen begleitet.

Diesen „kreuzgeschichteten Sanden“, die im Flämischen Tal 3 bis 4 m mächtig sind, lagern gelbliche, schwach lehmige Feinsande auf, die durch eine sehr feine Schichtung gekennzeichnet sind. Bisweilen haben sie fluviatilen Charakter, es treten aber auch echte Lehmschichten auf, die wie in Zelzate, kryoturbiert sein können. Die Fazies erinnert lebhaft an den „Alteren Flugdecksand I“ der Niederlande und wurde in Belgien als „Decksand 1" bezeichnet (PAEPE 1967)6). Stellenweise fanden sich hier pflanzliche Reste, wovon Salix herbacea zu erwähnen wäre (VANHOORNE). Neben zahlreichen kleinen Frostspalten innerhalb des „Decksands 1 “ kommt an der Oberkante eine überall zu verfolgende Steinsohle vor, die von großen Frostkeilen durchschnitten wird. Die Steinsohle kann trotz der großen Zahl windpolierter Feuersteine in eine mehr tonige, fluviatile Schicht übergehen. Sie erinnert lebhaft an die „Beuningen-Steinsohle“ der Niederlande (vAN DER HAMmeN et al. 1967), die dieselbe stratigraphische Position einnimmt. Oberhalb dieser „Steinsohle 3 mit großen Frostkeilen“ folgen homogene Sande äolischen Ursprungs. Es ist dies der „Decksand 2“.

In Zelzate (Abb. 5) wird der „Decksand 2“" von einer Reihe von etwa vier, 5 bis $10 \mathrm{~cm}$ mächtigen Torflagen überdeckt. Es ist hierdurch eine weitere Zeit mit relativ dich-

4), 5) Siehe Fußnote 3).

6) Der Name „Decksand“ ist schon seit längerer Zeit in Belgien eingebürgert (TAVERNIER 1943) und umfaßte ursprünglich alle hoch- und spätglazialen äolischen Sande. Letztere haben wir vor kurzem als "späten Decksand“ bezeichnet und zur gleichen Zeit den hochglazialen Decksand weiter untergeteilt. In stratigraphischer Hinsicht decken sich die alten und neuen Begriffe nicht immer (Paepe und Vanhoorne 1967). 
rer Vegetation nachgewiesen, was sich durch die ${ }^{14} \mathrm{C}$-Bestimmung von $12300 \pm 100$ Jahre vor heute (GrN-4782) bestätigt hat, die deutlich auf das Bølling-Interstadial hinweist. Schon vorher konnte dieser Horizont in Stabroek von R. VANHOoRne (De Coninck et al. 1966) erkannt und mit Hilfe der ${ }^{14} \mathrm{C}$-Methode datiert werden (12330; 12340 und 12460 J. v. h.). Es wurde der Name "S t a b r o e k-B o d e n " vorgeschlagen. Dieser Horizont ist öfters mit Kryoturbationen vergesellschaftet, die den überlagernden, homogenen, 4 bis $5 \mathrm{~m}$ mächtigen Sanden („später Decksand“) vollständig fehlen. Letztere können zweigeteilt sein und zwar durch eine dünne schwach humose Schicht, die nach den zahlreichen palynologischen Untersuchungen von R. VANHOORNE mit der Usselo-Schicht (Allerød) der Niederlande übereinstimmt. Es ist die Schicht, die öfters von kleinen Frostspalten durchschnitten wird und im allgemeinen nahe der Geländeoberfläche auftritt.

b. Das Lößgebiet (Abb. 4 )

Das Lößgebiet mit seinen zahlreichen Ziegeleigruben war ursprünglich am besten bekannt und diente als Ausgangspunkt für das Studium des Spätpleistozäns Belgiens (ManiL 1949, 1952; Gullentops 1954; Tavernier 1954). Sein welliges Relief, gekennzeichnet durch breite ebene Rücken und tiefe, wannenförmige Taleinschnitte, stammt fast vollkommen aus dem letzten Interglazial, das durch intensive Verwitterung und Bodenbildung charakterisiert ist. Diese Verwitterung, wofür F. Gullentops (1954) den Namen „R o c o u r t - B o d e n“ prägte, ist meistens als rotbraun gefärbter, stark toniger, geköpfter Textur-B-Horizont an der Oberkante der älteren Lößablagerungen zu sehen, die die tertiären, mesozoischen oder gar paläozoischen Schichten überdecken. Falls die älteren Lösse fehlen, scheint dies auch mit dem „Rocourt-Boden“ der Fall zu sein.

Der „Rocourt-Boden“ wird von einem grauen, heterogenen Lehm mit zahlreichen Fe u. Mn-Konkretionen überlagert.

Die Grenzschicht ist unregelmäßig, außerdem finden sich vereinzelt Frostkeile. An der Oberkante wird der graue Lehm humos und wurde früher von R. TAvernier $(1954,1957)$ als „horizon humifère" bezeichnet, während der Lehm als "limon gris à points noirs" angedeutet wurde; das Ganze wurde für Würm I gehalten. Wichtig ist aber, daß die Folge roter Boden - unregelmäßiger Kontakt - Lehm - humoser Boden, stark an jene von Zelzate erinnert; es handelt sich hier also um den Übergang vom Interglazial zum Frühglazial. Ein weiterer Hinweis ist die Tatsache, daß B. BASTIN (1967) auf Grund seiner Pollendiagramme in Rocourt und in gewisser Höhe auch in Tongrinne das Vorhandensein des Amersfoort- und des Brørup-Interstadials an der Unter- und Oberkante der humosen Schicht erkennen konnte. Weiter hatte R. PAEPE (1966) schon vorher auf die unverkennbare morphographische Ahnlichkeit der österreichischen und belgischen Lößprofile hingewiesen.

Wie im Decksandgebiet kann der erste Lehm seitlich in eine andere Fazies übergehen, nämlich in ziemlich grobes fluviatiles Material (Volkegem) oder sogar in einen basalen Kies (Racour). Auch hier sind die Namen "Lehme und Grobsande" und „Sand und Kies" ohne weiteres zutreffend. Es werden alle diese Schichten immer wieder an der Oberkante scharf abgegrenzt durch eine Reihe kleinerer Frostspalten und vereinzelte windgeschliffene Kiese (Steinsohle I mit kleinen Frostkeilen“).

Es folgt dann zuerst eine dünne, homogene gelbe Lößschicht, dann eine regelmäßige Wechsellagerung dünner sandiger und lehmiger Schichten mit kleinen Frostspalten und schließlich wieder eine dünne homogene Lehmschicht. Es ist deutlich, daß es sich, wenn wir von den reinen Lößlagen absehen, um ein Äquivalent der rhythmisch geschichteten „torfigen Lehmschichten“ von Zelzate und Gent handelt. Es fehlt hier aber der Torfanteil, und es wird daher von „Lehmschichten “ gesprochen. In derselben stratigraphischen Position wie der Zelzate-Boden tritt an der Oberkante dieser Schichten ein brauner Struktur-B-Horizont auf, der ebenfalls immer kryoturbat verformt ist. F. Gullentops be- 
zeichnet diesen Bodenrest als „K e s s e lt-B o d e n“. Die „Lehmschichten“ umfassen ungefähr die von Tavernier als Würm II gedeuteten Ablagerungen. F. Gullentops faßte alle Schichten oberhalb des Rocourt-Bodens und der humosen Basisschicht bis einschließlich der "Lehmschichten“ unter dem Begriff „Hesbayen“ zusammen. Dieser Komplex ist an der Oberkante meistens durch eine weitere Steinsohle, „Steinsohle 2 mit kleinen Frostkeilen", abgegrenzt.

Die Lößablagerungen oberhalb dieses Horizonts wurden von TAvernier ungefähr als Würm III, von F. Gullentops als „Brabant:en“ bezeichnet, aber nicht weiter gegliedert. Es handelt sich aber um ein mächtiges Lößpaket, das öfters sehr deutlich zweigeteilt ist und zwar durch eine Zone mit langen, nadelförmigen Frostkeilen („Steinsohle 3 rnit großen Frostkeilen“). Der Lehm unterhalb dieser Steinsohle ist der „Decklehm 1“, der höhere der „Decklehm 2“. Letztere Namen wurden grundsätzlich gewählt, um die lithostratigraphische Ubereinstimmung mit „Decksand 1“ bzw. "Decksand 2“ deutlich hervorzuheben (PAEPE und VANHOORNE 1967). In gewissen Fällen ist nur der letztere vertreten. Es fallen dann die Steinsohlen 2 und 3 zusammen und es treten große, breite Frostkeile auf, die tief in die älteren L.ößablagerungen vordrinsen. Die Ausbildung der Profile im niederländischen Lößgebiet (z. B. Sittard) ist durchaus gleichartig mit der hier behandelten Abfolge.

\section{c. Das Übergangsgebiet (Abb. 4)}

Das Übergangsgebiet bildet die geologische Übergangsform, ist aber auch in der Topographie zwischen der höher gelegenen Lehmregion am Nordrand der Ardennen und dem Decksandgeb:et im Norden eingeschaltet. Das Relief wird gekennzeichnet durch einen schnellen Wechsel von Rücken und Wannen, die fast unmerklich ineinander übergehen. Im Bereich der Rücken tritt der tertiäre Untergrund oft fast zu Tage, während er in den Wannen meistens tief unter die Pleistozänbedeckung abtaucht. Im Gegensatz zu den zwei vorigen Gebieten ist das Ubergangsgebiet als durch einen schnellen horizontalen Wechsel von dünnen und dicken Quartärdecken gekennzeichnet. Aber auch die Fazies der Deckschichten ändert sich stark, je nachdem, ob es sich um Rücken und Ebenen oder um Täler handelt. Ein Höhenunterschied von nur 2 bis 3 m genügt schon, um starke fazielle Anderungen he:vorzubringen.

In den Wannen lagern die letzteiszeitlichen Schichten meistens direkt dem Tertiär auf. In gewissen Fällen, wie z. B. in Rumbeke, findet sich also auch älterer Löß, außerdem ist eine mächtige eemzeitliche Torfschicht zwischengeschaltet.

Die letzieiszeitlichen Ablagerungen fangen in der Regel mit einem humosen oder sogar torfigen Horizont an, der sich den Wänden der Depressionen entlang nach oben fortsetzt. R. VANHoorne (1967) hat in Poperinge diesen Horizont pollenanalytisch als AmersfoortInterstadial datieren können. Außerhalb der Depressionen tritt der Torf zurück und wir finden einen humosen Horizont, der dann öfters einem roten Textur-B-Horizont aufliegt. In diesem Falle haben wir also Verhältnisse, wie sie uns schon mehrfach in den Löß- und Decksandgebieten begegnet sind. Nach dem Aufschluß in Warneton, wo sich dieser humose Horizont oberhalb des etwas vergleyten "Rocourt-Bodens“ besonders gut hervorhebt, wurde der Namen „W a r n e t o n-B od e n“ geprägt (PAePE 1963, 1964, 1967). Zeitlich gehört dieser also etwa in das Amersfoort-Interstadial.

Dann folgen Ablagerungen, die faziell den „Lehmen und Grobsanden“ anderer Stellen stark ähneln, während auch die „torfigen Lehmschichten“ mit ihren charakteristischen Sedimentationsrhythmen wieder auftreten (Poperinge, Rumbeke, Warneton). An der Basis wurde ein weiterer humoser Horizont gefunden, der "P o pering e-B o d e n“, der auf $45600 \pm 1500$ Jahre v. h. datiert wurde (GrN-4856). Es sei hier an Zelzate erinnert, wo gleichfalls unmittelbar oberhalb des roten Bodens zwei torfig-humose Horizonte auftraten. In Rumbeke findet sich in den „tonigen Lehmschichten “ noch eine weitere 
kryoturbate Humusschicht unterhalb des „Kesselt-Zelzate-Bodens“, wiederum ähnlich wie in Zelzate. In Hoboken wurde dieser Horizont ebenfalls erkannt und mit $32490 \pm 440$ J. v. h. datiert (GrN-4781). Obwohl diese Datierung sich weniger gut mit den niederländischen Daten vergleichen läßt und daher weitere Proben untersucht werden sollten, scheint sie doch die Zwischenstellung zwischen „Kesselt-Zelzate“ und „Poperinge“ zu bestätigen. Es ist damit eine weitere Zeit mit günstigeren Vegetationsbedingungen bezeugt. Vorläufig wurde dieser Horizont mit dem Namen „H o b o k e n - B o d e n“ belegt.

Es zeigt sich also, daß sowohl im Decksandgebiet wie im Übergangsgebiet mehrere Bodenbildungen innerhalb des Komplexes der „torfigen Lehmschichten“ auftreten können. Bis jetzt wurden sie in den stratigraphisch äquivalenten "Lehmschichten “ der Lößregion nicht mit Sicherheit wiedergefunden. Südlich von Mons finden sich aber in mehr als $8 \mathrm{~m}$ mächtigen Lehmschichten mehrere Böden, die mit den erwähnten Horizonten übereinstimmen können (PAEPE, unveröff. 1968).

Vom geomorphologischen Standpunkt aus sei darauf hingewiesen, daß die Wannen mit „Lehmen und Grobsanden“ und „torfigen Lehmschichten“ zur Zeit der „Kesselt-Bodenbildung" eingeebnet waren. In der dann geformten flachen Landschaft wurden Decklehme und Decksande abgelagert, teilweise auch gleichzeitig die „kreuzgeschichteten Sande“. In Poperinge z. B. geht ein reiner Lehm im Abstand von weniger als 0,20 $\mathrm{m}$ in grobe, kreuzgeschichtete Sande über. Auch ist die Fazies unter der „Steinsohle 3“ meistens komplex und es findet sich „Decklehm 1 “ neben und über „Decksand 1“. Die sandige Fazies überwiegt in den Schichten oberhalb der Steinsohle einschließlich der spätglazialen Schichten. Es zeigt sich also ein Umschwung von überwiegend lehmiger Sedimentation in eine überwiegend sandige.

\section{d. Diskussion}

Wie sich aus den Beschreibungen und Abb. 4 ergibt, finden sich im Prinzip die verschiedenen lithostratigraphischen Einheiten in allen Gebieten wieder, wenn auch die Ausbildung verschieden sein kann. Es können daher das Decksandgebiet und das Lößgebiet verknüptt werden. Im Decksandgebiet ist die letzteiszeitliche Folge unterlagert von marinen Ablagerungen, die u. a. auf Grund des Vorkommens von Tapes senescens var. eemiensis als gesichertes Eem-Interglazial im Sinne der nordwesteuropäischen Stratigraphie angesprochen werden können (PAEPE 1965). Hiermit haben wir aber einen Pfeiler für die gesamte spätpleistozäne Stratigraphie Belgiens und es wird auch deutlich, daß die nordwesteuropäische Terminologie der alpinen vorgezogen werden sollte; es sollte daher von Weichsel und nicht von Würm gesprochen werden. Die Tatsache, daß im Decksandgebiet der Rocourt-Boden mit den marinen Eem-Schichten in Verbindung gebracht werden konnte, brachte weiterhin mit sich, daß diese Terminologie auch im Lößgebiet verwendet werden konnte. Das belgische Lößgebiet hat zwar mehr morphostratigraphische Ahnlichkeit mit dem zentraleuropäischen (PAePE 1966) als mit dem Decksandgebiet, aber es gibt doch keine Terrasse oder Moräne, die den direkten Zusammenhang mit dem alpinen Raum belegen könnte.

Weiter können in den Lößprofilen meistens drei Einheiten unterschieden werden. Früher war dies ein Grund für die Verwendung der SoERgelschen Klassifikation (Würm I, II, III). Eine Zweigliederung ist aber besser zu verteidigen, wie sie von PAEPE und VANHOORNe (1967) vertreten wurde. Als erster Zyklus wurden zum Pleniglazial A mit kalt-feuchtem Klima die folgenden Einheiten gerechnet: „Sande und Kiese“, „Lehme und Grobsande“, „Steinsohle 1“, „torfige Lehmschichten“, der „Kesselt-Zelzate-Boden“. Der zweite Zyklus besteht aus Schichten des Pleniglazials B, die unter kalt-trockenen Klimabedingungen gebildet wurden. Hierzu gehören: die „kreuzgeschichteten Sande“ und die Decklehme und Decksande mit der "Steinsohle 3“. Weiter gehören dazu die sandigen und torfigen spätglazialen Schichten. 


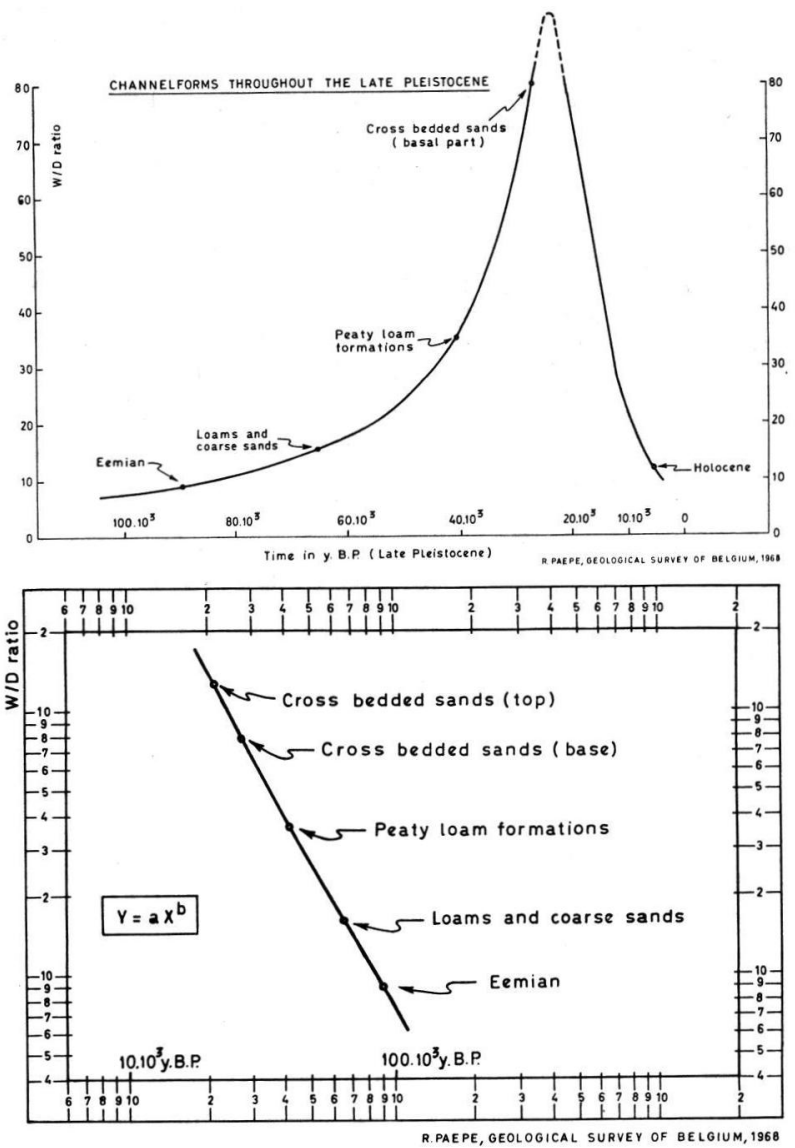

Abb. 6. Entwicklung der Breiten-Tiefen-Verhältnisse der spätpleistozänen Flußbettform in Belgien.

Beide Zyklen fangen mit groben Sedimenten an und enden in Schichten mit schneller rhythmischer Sedimentation: „torfige Lehmschichten“ bzw. „Decksand 2" und spätglaziale Ablagerungen. In beiden Zyklen tritt ein Kältemaximum auf, das an der Bildung einer von Frostkeilen begleiteten Steinsohle erkennbar ist. Letztere vertritt u. E. einen stratigraphischen Hiatus und deutet in klimatischer Hinsicht auf eine lange Zeit intensiver Kälte. Der Begriff „Polarwüste“, der in den Niederlanden dafür eingeführt wurde (vaN DER HAmmen et al. 1967), scheint uns sehr zutreffend, um so mehr, da es sich um eine außerordentlich flache und weitverbreitete Steinsohle handelt.

Es treten aber auch im „Pleniglazial A“ dieser Autoren zwei Hauptphasen mit auffallenden interstadialen Merkmalen auf. Es sind dies die Phase der "Lehme und Grobsande" mit dem Warneton-Boden und die "torfigen Lehmschichten" mit nicht weniger als drei Böden: Poperinge (rund 45000 Jahre vor heute), Hoboken (rund 33000 ? Jahre vor heute) und $\mathrm{K}$ esse $1 \mathrm{t}-\mathrm{Ze} \mathrm{l} \mathrm{z}$ a t e (rund 28000 Jahre vor heute).

$\mathrm{Da}$ aber während der Bildung des W a r n e to n-Bodens die Landschaft noch zeitweise bewaldet war, im Gegensatz zur waldlosen Vegetation der jüngeren Interstadiale, kann auf paläobotanischer Grundlage dieser Komplex als frühglazial abgetrennt werden, analog dem Vorgehen von W. H. ZAGWIJN (1961) in den Niederlanden. Weitere Ver- 
gleiche mit der niederländischen Gliederung sollen in einem nächsten Paragraph behandelt werden.

Wenn die Weichsel-Kaltzeit auch in verschiedene Phasen kälteren und wärmeren Klimas zu unterteilen ist, so kann nicht verneint werden, daß im Verlaufe dieser Stufe das Klima im allgemeinen immer kälter und trockener geworden ist. Wir möchten dies anhand der Abb. 6 belegen. Das Breiten-Tiefen-Verhältnis der verschiedenen Flußbettformen des Spätpleistozäns ist hier der Ordinate zugeordnet, die Zeit der Abszisse. Die erhaltene Kurve ist eine logarithmische, die auf doppeltlogarithmischem Papier als gerade Linie abgebildet wird. Da der Flußbettquerschnitt u. a. vom Abfluß und der Menge der Bodenlast abhängt, ist ein Zusammenhang mit Temperatur und Feuchtigkeit des jeweiligen Klimas gegeben. Bei großem Abfluß und relativ geringer Sedimentmenge schneiden sich die Rinnen stark ein. Flache Rinnen bilden sich bei geringem Abfluß und großer Bodenlast. Der Übergang von der einen in die andere Form wird dann stattfinden, wenn das Klima sich von feucht-warm in trocken-kalt abwandelt. Starke Solifluktion ist vor allem ein wesentlicher Beitrag zur Erhöhung der Bodenlast der Flüsse während der Kaltzeiten. Betrachten wir die Klimaevolution der Weichsel-Kaltzeit von diescm Gesichtspunkt aus, so ergibt sich doch eine mehr kontinuierliche Klimaevolution bis zum Kältemaximum am Ende dieser Zeitspanne, als sich aus der Stratigraphie der Ablagerungen ableiten ließ.

\section{Schlußfolgerungen}

Vergleichen wir schließlich kurz die im vorhergehenden Abschnitt behandelten Gliederungen der Niederlande und Belgiens, so zeigt sich eine auffällige Übereinstimmung. Leider ist die Zahl der ${ }^{14} \mathrm{C}$-Daten in Belgien noch nicht sehr groß und man kann nur hoffen, daß in Zukunft weitere solcher Daten die hier gegebenen Korrelationen besser untermauern werden.

Wie schon hervorgehoben wurde, können in beiden Gebieten die eeminterglazialen Schichten als stratigraphischer Leithorizont verwendet werden. Es lassen sich die frühglazialen Schichten, die in den Niederlanden schon länger erkannt wurden, auch im südlichen Bereich in verschiedener Ausbildung abtrennen, so u. a. als „Warneton-Boden“.

In der Arbeit von PAepe und Vanhoorne (1967) wurden diese Schichten noch als "Pleniglazial A“ angesprochen, u. a. auf Grund des Vorkommens starker kryoturbater Störungen und wegen des Fehlens von Sedimenten des „Unteren Pleniglazials“. Solche Strukturen sind aber neuerdings auch in den Niederlanden im frühglazialen Bereich beobachtet worden (Abb. 2) und es ist damit kein Grund vorhanden, den korrelaten belgischen Schichten ein frühglaziales Alter abzusprechen. Belege für das Vorhandensein des Amersfoort- und des Brørup-Interstadials in Belgien beruhen vor allem auf pollenanalytischen Daten, die oben diskutiert wurden. Ein weiterer Ausbau dieses Datenmaterials ist aber notwendig.

Im pleniglazialen Bereich sind einige lithostratigraphische Hauptmerkmale zu verzeichnen, die beiden Regionen gemeinsam sind. Es ist dies an erster Stelle das Vorhandensein zweier Hauptfrostkeilzonen und Steinsohlen - die eine (Unteres Pleniglazial, „Steinsohle 1") an der Basis, die andere (Beuningen-Steinsohle, "Steinsohle 3“) im oberen Teil des Pleniglazials. Es deutet dies darauf hin, daß es zwei Zeiten maximaler Kälte mit Polarwüstenklima gegeben hat. Auf Grund der niederländischen Datierungen gehört die erste in die Zeit vor 50000 Jahre vor heute, die zweite in die Zeit zwischen 26000 und 14000 Jahre vor heute.

Zwischen diese beiden Kältemaxima fällt die Zeit des Mittleren Pleniglazials, ungefähr zwischen 50000 und 28000 Jahre vor heute. Im allgemeinen hebt sich diese Phase heraus durch das Auftreten von lehmigen und torfigen Ablagerungen, die teilweise mit Sandschichten abwechseln. Wie aus den niederländischen Beobachtungen hervorgeht, weist 
dieser Abschnitt ein Tundrenklima auf und es lassen sich mit Sicherheit zwei Interstadiale erkennen ("D e nekam p“ um 30000 J. v. h. und „Hengelo" um 38000 herum). Ein drittes Interstadial („Moershoof d“) kann um etwa $45000 \mathrm{~J}$. v. h. vermutet werden.

In Belgien findet sich eine wichtige Bodenbildung an der Oberkante dieses Komplexes, $\operatorname{der} \mathrm{Zelz}$ a te-Kesse1t-Boden, dessen morphologische Stellung im Lößbereich eine Korrelation mit dem „Paudorf-Stillfried-B“-Komplex des ostalpinen Lößgebietes nahelegt. Die ${ }^{14} \mathrm{C}$-Datierung in Zelzate befindet sich damit in Übereinstimmung, bestätigt aber zur gleichen Zeit die Gleichaltrigkeit mit dem Denekamp-Interstadial der Niederlande. Es finden sich unterhalb dieses Bodens noch bisweilen zwei weitere - der $\mathrm{H}$ o b ok e n Boden und der Popering e-Boden. Letzterer wurde auf rund 45000 Jahre datiert und es ist damit eine Zeitgleichheit mit dem Moershoofd-Interstadial der Niederlande gegeben. Außerdem tritt dieser Boden, vor allem im Ubergangsgebiet immer an der Unterkante der „torfigen Lehmschichten“ auf. In lithostratigraphischer Hinsicht scheint dieses Interstadial in Belgien also gesichert zu sein. Der stratigraphischen Stellung nach muß der Hoboken-Boden mit dem Hengelo-Interstadial übereinstimmen. Das vorhandene ${ }^{14} \mathrm{C}$ Datum beweist dies leider nicht; es werden daher weitere Untersuchungen notwendig sein.

Das Obere Pleniglazial der Niederlande ist gekennzeichnet durch sehr auffällige Flugdecksande und die Beuningen-Steinsohle. Das „Pleniglazial B“ in Belgien nach PAEPE und VANHOORNE (1967) ist vor allem im Decksandgebiet durchaus ähnlich und es ist angebracht, weiterhin auch dort den Terminus „Oberes Pleniglazial“ zu verwenden. Es ist dies die Zeit der größten Kälte und außerdem der größten Trockenheit der ganzen Weichselzeit (Abb. 6). Im Lößbereich findet jetzt reine Lößbildung statt. Der Beuningen-Horizont — „Steinsohle 3“ - zeichnet sich aber auch dort deutlich ab.

Die Stratigraphie des Spätglazials der beiden Regionen ist ohne weiteres vergleichbar und durch eine größere Zahl der ${ }^{14} \mathrm{C}$-Daten belegt. Im Lößbereich fehlen Schichten dieser Zeit vermutlich vollständig.

\section{Schrifttum}

Aleksandrova, V. D.: Some regularities in the distribution of the vegetation in the arctic toundra. Arctic 13, 147-162, Ottawa 1960.

Andersen, S. T., Hl. De Vries \& W. H. Zagwijn: Climatic change and radiocarbon dating in the Weichselian Glacial of Denmark and the Netherlands. Geol. en Mijnb. 39, 38-42, 's-Gravenhage 1960.

Averdieck, F. R.: Die Vegetationsentwicklung des Eem-Interglazials und der Frühwürm Interstadiale von Odderade-Schleswig-Holstein. Fundamenta 2 (i. Dr.).

Bastin, B.: Pollendiagramm Tongrinne I. In: Meeting of the „Sub-commission for Loess-stratigraphy" in Belgium, 1967. Guidebook, compiled by R. PAEPE. Rocourt I, Ibidem, Excursion N. E. Belgium (Gullentops, Bastin), 1967. - - Pflanzengeographische Probleme der offenen Vegetation Europas während der letzten Eiszeit. Ber. Deutsch. Bot. Ges. 80, 10, 697-704, Berlin 1968.

BüDeL, J.: Die Klimaphasen der Würmeiszeit. Die Naturwiss. 37, 438-449, Berlin 1950.

ConinCK, Fr. DE, P. Greguss \& R. Vanhoorne: La superposition des dépôts tourbeux datant des oscillations Allerød et Bølling à Stabroek (Belgique). Pédologie XVI, 3, 293-308, Gand 1966.

Gross, H.: Das Göttweiger Interstadial, ein zweiter Leithorizont der letzten Vereisung. Eiszeitalt. u. Gegenw. 7, 87-101, Öhringen 1956. - - Die bisherigen Ergebnisse von C14-Messungen und paläontologischen Untersuchungen für die Gliederung und Chronologie des Jungpleistozäns in Mitteleuropa und den Nachbargebieten. Eiszeitalt. u. Gegenw. 9, 155-187, OOhringen 1958. - - Das Mittelwürm in Mitteleuropa und angrenzenden Gebieten. Eiszeitalt. u. Gegenw. 15, 187-198, OOhringen 1964.

Gullentops, F.: Contributions à la chronologie du Pleistocène et des formes de relief en Belgique. Mém. Inst. Géol. de Louvain, XVIII, 125-252, Louvain 1954. - - Stratigraphie du Pleistocène supérieur en Belgique. Geol. en Mijnb., N. S. 39, 7, 305, 's-Gravenhage 1957. 
Hammen, T. van DER: Late Glacial flora and periglacial phenomena in the Netherlands. Leidse Geol. Mededel. 17, 71-183, Leiden 1951. - - Dating and correlation of periglacial deposits in Middle and Western Europe. Geol. en Mijnb., N. S. 14, 9, 238-336, 's-Gravenhage 1952.

Hammen, T. van Der, G. C. MaArleveld, J. C. Vogel \& W. H. Zagwijn: Stratigraphy, climatic succession and radiocarbon dating of the Last-Glacial in the Netherlands. Geol. en Mijnb. 46, 3, 79-95, 's-Gravenhage 1967.

ManiL, G.: Le Quaternaire des environs de Gembloux. La tranchée de Mazy. Bull. Soc. belge de Géol. LVIII, 1, 139-152, Brüssel 1949. - - Quelques considérations générales sur la stratigraphie quaternaire et la pédogénèse à propos de la description de trois coupes de loess (Hesbaye Gembloutoise). Ann. Soc. Géol. de Belgique LXXXV, 153-167, Liége 1952.

PAEpe, R.: Bouw en Oorsprong van de Vlakte van de Leie. - Doctors thesis, 225 S., 1963. - Les dépôts quaternaires de la plaine de la Lys. Bull. Soc. belge de Géol. LXXIII, 3, 327-365, Brüssel 1964. - - On the presence of Tapes senescens in some borings of the coastal plain and the Flemish Valley of Belgium. Bull. Soc. belge de Géol. LXXIV, 2, 1-5, Brüssel 1964. - - Comparative stratigraphy of Würm loess deposits in Belgium and Austria. Bull. Soc. belge de Géol. LXXV, 2, 203-216, Brüssel 1964.

Paepe, R. \& R. VAnhoorne: The Stratigraphy and Palaeobotany of the Late Pleistocene in Belgium. Memoir Nr. 8, Cartes géol. et min. de la Belgique, 96 S., 1967.

TAvernier, R.: De kwartaire afzettingen van België. Nat. Wet. Tijdschrift 25, 121-137, Gent 1943. - - L'évolution du Bas-Escaut au Pleistocène supérieur. Bull. Soc. belge de Géol. LV, 1, 106-125, Brüssel 1946. - - Le Quaternaire. Prodrome d'une description géologique de la Belgique, 555-589, Liège 1954.

TAvernier, R. \& J. DE Heinzelin: Chronologie du Pleistocène supérieur, plus particulièrement en Belgique. Geol. en Mijnb., N. S. 7, 306-309, 's-Gravenhage 1957.

Vogel, J. C. \& W. H. Zagwijn: Groningen Radiocarbon dates VI. Radiocarbon 9, 63-106, Yale 1967.

Vries, Hl. de, G. W. Barendsen \& H. T. Waterbolk: Groningen Radiocarbon dates II. Science 127, 129-137, Yale 1958.

ZaGwiJn, W. H.: Vegetation, Climate and Radiocarbon datings in the Late Pleistocene of the Netherlands. Part I. Eemian and Early Weichselian. Med. Geol. Sticht., N. S. 14, 15-45, 's-Gravenhage 1961.

Manuskr. eingeg. 21. 5. 1968.

Anschriften der Verf.: Dr. W. Zagwijn, Rijks Geologische Dienst, Spaarne 17, Haarlem, Niederlande und Dr. R. Paepe, Aardkundige Dienst van België/Service Géologique de Belgique, Jennerstraat 13, Brüssel, Belgien. 